\title{
Electromagnetic Energy, Momentum, and Angular Momentum in an Inhomogeneous Linear Dielectric
}

\author{
Michael E. Crenshaw and Thomas B. Bahder \\ US Army Aviation and Missile Research, Development, \\ and Engineering Center, Redstone Arsenal, AL 35898, USA
}

(Dated: October 31, 2018)

\begin{abstract}
In a previous work, Optics Communications 284 (2011) 2460-2465, we considered a dielectric medium with an anti-reflection coating and a spatially uniform index of refraction illuminated at normal incidence by a quasimonochromatic field. Using the continuity equations for the electromagnetic energy density and the Gordon momentum density, we constructed a traceless, symmetric energy-momentum tensor for the closed system. In this work, we relax the condition of a uniform index of refraction and consider a dielectric medium with a spatially varying index of refraction that is independent of time, which essentially represents a mechanically rigid dielectric medium due to external constraints. Using continuity equations for energy density and for Gordon momentum density, we construct a symmetric energy-momentum matrix, whose four-divergence is equal to a generalized Helmholtz force density four-vector. Assuming that the energy-momentum matrix has tensor transformation properties under a symmetry group of space-time coordinate transformations, we derive the global conservation laws for the total energy, momentum, and angular momentum.
\end{abstract}

\section{INTRODUCTION}

Starting with the hydrodynamic continuity equation, Umov [1] obtained an expression for energy continuity in a continuous spatial flow of an electromagnetic field in 1874 [2-4]. A decade later, Poynting [2] derived a similar energy continuity equation as a general theorem of the macroscopic Maxwell equations. Poynting's theorem is generally preferred because it can be derived directly from the macroscopic Maxwell equations

$$
\begin{gathered}
\nabla \times \mathbf{B}-\frac{n^{2}}{c} \frac{\partial \mathbf{E}}{\partial t}=0 \\
\nabla \times \mathbf{E}+\frac{1}{c} \frac{\partial \mathbf{B}}{\partial t}=0 \\
\nabla \cdot\left(n^{2} \mathbf{E}\right)=0 \\
\nabla \cdot \mathbf{B}=0
\end{gathered}
$$

of classical continuum electrodynamics. Here, the Maxwell equations are written in Heaviside-Lorentz units for a nonmagnetic linear medium in the absence of free charges and currents. We have assumed that the index of refraction, $n=n(\mathbf{r})$, depends on position, occupies a finite region of 3-dimensional space, and is independent of time. Poynting's theorem can be derived by subtracting the scalar product of the Maxwell-Ampère law, Eq. (1.1), with $\mathbf{E}$ from the scalar product of Faraday's law, Eq. (1.2), with $\mathbf{B}$ to obtain

$$
\begin{gathered}
\frac{n^{2}}{c} \frac{\partial \mathbf{E}}{\partial t} \cdot \mathbf{E}+\frac{1}{c} \frac{\partial \mathbf{B}}{\partial t} \cdot \mathbf{B} \\
+(\nabla \times \mathbf{E}) \cdot \mathbf{B}-(\nabla \times \mathbf{B}) \cdot \mathbf{E}=0 .
\end{gathered}
$$

Upon application of a vector identity and the definition of the energy density,

$$
\rho_{e}=\frac{1}{2}\left(n^{2} \mathbf{E}^{2}+\mathbf{B}^{2}\right)
$$

the preceding equation becomes Poynting's theorem

$$
\frac{\partial \rho_{e}}{\partial t}+\nabla \cdot c(\mathbf{E} \times \mathbf{B})=0
$$

and defines Poynting's energy flux vector $\mathbf{S}_{P}=c(\mathbf{E} \times \mathbf{B})$.

In a previous work [5], we considered a dielectric medium with an index of refraction that was timeindependent, spatially uniform, and covered with a thin gradient-index antireflection coating. For this case, we showed that the energy

$$
U=\int_{\sigma} \frac{1}{2}\left(n^{2} \mathbf{E}^{2}+\mathbf{B}^{2}\right) d v
$$

and the Gordon [6] momentum

$$
\mathbf{G}_{G}=\int_{\sigma} \frac{1}{c}(n \mathbf{E} \times \mathbf{B}) d v
$$

are the conserved electromagnetic quantities with integration performed over all space $\sigma$. We constructed the corresponding traceless, symmetric energy-momentum tensor, whose four-divergence provided the continuity equations for the energy and momentum densities of the closed system. This result incorporated a condition of $\nabla n / n \ll 1 / \lambda$ corresponding to unimpeded flow in the absence of external forces.

In the current work, we consider the case of an inhomogeneous dielectric medium in which the condition $\nabla n / n \ll 1 / \lambda$ no longer holds. The assumption that the index of refraction is independent of time necessarily implies that the dielectric medium is mechanically rigid [7] and that no momentum is transferred to the dielectric 
medium. In other words, at each spatial point, there is effectively an external field that acts as a constraint that holds the dielectric in place. Therefore, the system that we are considering is not a closed system and the continuity equations and global conservation equations will reflect this feature. The external field that acts as a constraint is not a field that we impose, instead it arises as a result of the way in which we arrange terms in the continuity equations. We construct the energy and momentum continuity equations and find that the spatial gradient of the refractive index appears in the continuity equations in a generalized Helmholtz force density. This generalized Helmholtz force density is a spatial and time-varying field that provides the constraint for the dielectric medium to be mechanically rigid. We then write the continuity equation as a four divergence of a symmetric energy-momentum matrix. Assuming that the energy-momentum matrix has tensor transformation properties under a symmetry group of space-time coordinate transformations, we apply the four-dimensional divergence theorem to derive global conservation laws for the total energy, momentum, and angular momentum.

\section{ENERGY AND MOMENTUM CONTINUITY EQUATIONS}

The energy-momentum tensor is a concise way to represent the local continuity of energy and momentum of a field. While simple in concept, the form of the energy-momentum tensor for the electromagnetic field in a dielectric has been at the center of the centurylong Abraham-Minkowski controversy [8]. The tensor that was proposed by Minkowski [9] in 1908 is not diagonally symmetric, a fact that is adverse to conservation of angular momentum [10]. Abraham [11] subsequently proposed a symmetric tensor at the expense of a phenomenological force. The disagreeable properties of the Minkowski and Abraham energy-momentum tensors are manifestations of underlying conservation issues: neither the Minkowski momentum nor the Abraham momentum is conserved. In this section, we derive the energy and momentum continuity equations for an inhomogeneous dielectric and construct the corresponding tensor continuity equation and energy-momentum tensor. In the following section, we will obtain the globally conserved quantities - the total energy, the total momentum, and the total angular momentum system, from the energy momentum tensor.

Starting from the Maxwell Eqs.(1.1)-(1.4), we use the continuity of the electromagnetic energy density and the Gordon momentum to obtain a symmetric stress-energy tensor. We begin with the temporal derivatives of the energy density and the Gordon momentum density,

$$
\frac{n}{c} \frac{\partial}{\partial t} \frac{1}{2}\left(n^{2} \mathbf{E}^{2}+\mathbf{B}^{2}\right)=\frac{n}{c} \frac{\partial(n \mathbf{E})}{\partial t} \cdot n \mathbf{E}+\frac{n}{c} \frac{\partial \mathbf{B}}{\partial t} \cdot \mathbf{B}
$$

$$
\frac{n}{c} \frac{\partial}{\partial t}(n \mathbf{E} \times \mathbf{B})=\frac{n}{c} \frac{\partial(n \mathbf{E})}{\partial t} \times \mathbf{B}+n \mathbf{E} \times \frac{n}{c} \frac{\partial \mathbf{B}}{\partial t} .
$$

The Gordon momentum in Eq. (2.2) has been scaled by $c$ so that the equations are in the same units. Next, we apply the vector identity

$$
\nabla \times(\psi \mathbf{a})=\nabla \psi \times \mathbf{a}+\psi \nabla \times \mathbf{a}
$$

to Faraday's law and write the macroscopic Maxwell equations, Eqs. (1.1) and (1.2), as

$$
\begin{gathered}
\frac{n}{c} \frac{\partial(n \mathbf{E})}{\partial t}=\nabla \times \mathbf{B} \\
\frac{n}{c} \frac{\partial \mathbf{B}}{\partial t}=-\nabla \times(n \mathbf{E})+\frac{\nabla n}{n} \times n \mathbf{E} .
\end{gathered}
$$

The variant form of Maxwell's equations, Eqs. (2.4) and (2.5), are mathematically equivalent to the original versions, Eqs. (1.1) and (1.2), respectively. Substituting the Maxwell equations, Eqs. (2.4) and (2.5), into Eqs. (2.1) and (2.2), we produce the energy continuity equation

$$
\frac{n}{c} \frac{\partial \rho_{e}}{\partial t}+\nabla \cdot(n \mathbf{E} \times \mathbf{B})=\frac{\nabla n}{n} \cdot(n \mathbf{E} \times \mathbf{B})
$$

and the (Gordon) momentum continuity equation

$$
\begin{gathered}
\frac{n}{c} \frac{\partial}{\partial t}(n \mathbf{E} \times \mathbf{B})+\nabla \cdot \mathbf{W}+n \mathbf{E}(\nabla \cdot n \mathbf{E})= \\
\left(n \mathbf{E} \times \frac{\nabla n}{n}\right) \times n \mathbf{E} .
\end{gathered}
$$

Here, we have used Eq. (1.3) and the definition of the Maxwell stress tensor [10, 12.

$$
W_{i j}=\left(-n E_{i} n E_{j}-B_{i} B_{j}+\frac{1}{2}(n \mathbf{E} \cdot n \mathbf{E}+\mathbf{B} \cdot \mathbf{B}) \delta_{i j}\right) .
$$

We can write the energy continuity equation, Eq. (2.6), and momentum continuity equation, Eq. (2.7), as the matrix differential equation

$$
\bar{\partial}_{\beta} T^{\alpha \beta}=f^{\alpha}
$$

with summation over repeated indices. The quantities that appear in Eq. (2.9) are a four-divergence operator

$$
\bar{\partial}_{\alpha}=\left(\frac{n}{c} \frac{\partial}{\partial t}, \partial_{x}, \partial_{y}, \partial_{z}\right),
$$

the array

$$
T^{\alpha \beta}=\left[\begin{array}{cccc}
\rho_{e} & c g_{\mathrm{G}_{1}} & c g_{\mathrm{G}_{2}} & c g_{\mathrm{G}_{3}} \\
c g_{\mathrm{G}_{1}} & W_{11} & W_{12} & W_{13} \\
c g_{\mathrm{G}_{2}} & W_{21} & W_{22} & W_{23} \\
c g_{\mathrm{G}_{3}} & W_{31} & W_{32} & W_{33}
\end{array}\right],
$$


and a generalized force density four-vector

$$
f^{\alpha}=\left(\nabla n \cdot(\mathbf{E} \times \mathbf{B}),-\mathbf{f}_{H}\right)
$$

where

$$
\mathbf{f}_{H}=-n E^{2} \nabla n+2 n \mathbf{E}(\mathbf{E} \times \nabla n)+n^{2} \mathbf{E}(\nabla \cdot \mathbf{E}) .
$$

Using Eq. (1.3), the last two terms on the right side of Eq. 2.13) cancel, so that

$$
\mathbf{f}_{H}=-n E^{2} \nabla n
$$

in the absence of free charges.

The array in Eq. (2.11) appears to have the properties of an energy-momentum tensor. By construction, the operator defined in Eq. (2.10) applied to the rows of the array in Eq. (2.11) generates continuity equations for the electromagnetic energy and the momentum. The same operation applied to the columns

$$
\bar{\partial}_{\alpha} T^{\alpha \beta}=f^{\beta}
$$

generates the same continuity equations by symmetry

$$
T^{\alpha \beta}=T^{\beta \alpha} .
$$

The array has a vanishing trace

$$
T_{\alpha}^{\alpha}=0
$$

corresponding to massless particles [10, 12].

The new feature of this result is the appearance of the four-vector $f^{\alpha}$ in the continuity equation (2.9). The appearance of the four-vector $f^{\alpha}$ in the divergence of the stress energy tensor in Eq. (2.9) is a result of the fact that the system is not closed. When the gradient of the index of refraction is non-zero, there is a back-action on the field altering its spatial properties, so the field does not experience "unimpeded flow". It is possible to define an effective stress-energy tensor that takes into account the back-action on the field by introducing

$$
\partial_{\alpha} t^{\alpha \beta}=f^{\beta} \text {. }
$$

The continuity of energy and momentum can then be expressed by

$$
\partial_{\alpha}\left(T^{\alpha \beta}+t^{\alpha \beta}\right)=0
$$

where the tensor $t^{\alpha \beta}$ contains the influence of the inhomogeneity of the material and is zero for homogeneous dielectrics.

\section{GLOBAL CONSERVATION EQUATIONS}

If we assume that $T^{\alpha \beta}$ transforms as a tensor under some symmetry group of space-time coordinate transformations, then we can apply the four-dimensional divergence theorem to obtain global conservation equations.
Integrating Eq. (2.9) over a four-volume, $d \Omega=c d \bar{t} d^{3} x \equiv$ $c d \bar{t} d v$ (where $\bar{t}=t / n$ ), between hypersurfaces [10] of constant time at $\bar{t}_{1}=t_{1} / n$ and $\bar{t}_{2}=t_{2} / n$, we have

$$
\int_{\bar{t}_{1}}^{\bar{t}_{2}} \bar{\partial}_{\beta} T^{\alpha 0} c d \bar{t} d v=\int_{\bar{t}_{1}}^{\bar{t}_{2}} f^{\alpha} c d \bar{t} d v
$$

where the integrals over $d v=d^{3} x$ are 3 -dimensional volume integrals over the volume containing the field. Applying the four-divergence theorem results in

$$
\int\left(T^{\alpha 0}\left(t_{2}\right)-T^{\alpha 0}\left(t_{1}\right)\right) d v=c \int \frac{1}{n} f^{\alpha} d t d v .
$$

When $\alpha=1,2,3$, Eq. (3.2) reduces to

$$
\mathbf{G}\left(t_{2}\right)-\mathbf{G}\left(t_{1}\right)=\int E^{2} \nabla n d t d v
$$

where $\mathbf{G}(t)$ is the Gordon momentum at time $t$, and the time integration is between $t=t_{1}$ and $t=t_{2}$. Equation (3.3) shows that the Gordon momentum of the field is not constant, i.e., there is a source or sink of momentum provided by the external constraint field $\mathbf{f}_{H}$. For the case $\alpha=0$, Eq. (3.2) gives

$$
\int\left(\rho_{e}\left(t_{2}\right)-\rho_{e}\left(t_{1}\right)\right) d v=\int \frac{\nabla n}{n} \cdot(\mathbf{E} \times \mathbf{B}) d t d v
$$

where $\rho_{e}$ is given by Eq. (1.6). The left side of Eq. (3.4) is the difference in total energy at two different times, $U\left(t_{2}\right)-U\left(t_{1}\right)$. We see that a non-zero spatial gradient in the index means that the field can gain or loose energy. The results given in Eqs. (3.3) and (3.4) assume that the matrix $T^{\alpha \beta}$ in Eq. (2.9) transforms as a tensor under some symmetry group of space-time coordinate transformations. Note that when $\nabla n=0$, Eqs. (3.3) and (3.4) show that the Gordon momentum $\mathbf{G}(t)$ and the energy $U(t)$ are constants.

In recent years, there is intense interest in angular momentum carried by the electromagnetic field, see the recent review and references cited therein [13, 14]. However, the angular momentum carried by the electromagnetic field in a dielectric environment is no less unsettled than the linear momentum case [15]. We can define the four-tensor of angular momentum density in terms of our energy-momentum tensor as [10]

$$
m^{\alpha \beta \gamma}=\frac{1}{c}\left(x^{\alpha} T^{\gamma \beta}-x^{\beta} T^{\gamma \alpha}\right)=-m^{\beta \alpha \gamma} .
$$

Continuity of angular momentum is given by

$$
\bar{\partial}_{\gamma} m^{\alpha \beta \gamma}=\frac{1}{c}\left(x^{\alpha} f^{\beta}-x^{\beta} f^{\alpha}\right) .
$$

The divergence of $m^{\alpha \beta \gamma}$ is not zero, thereby indicating that there is a source or sink of angular momentum density, due to the gradient of the index of refraction.

Once again, we assume that there exists a symmetry group of space-time coordinate transformations, so that 
$m^{\alpha \beta \gamma}$ is a tensor. As above, we can then use the fourdivergence theorem to obtain

$$
\int\left(m^{\alpha \beta 0}\left(t_{2}\right)-m^{\alpha \beta 0}\left(t_{1}\right)\right) d v=\int \frac{1}{n}\left(x^{\alpha} f^{\beta}-x^{\beta} f^{\alpha}\right) d t d v
$$

where the time integral is between the two times $t_{1}$ and $t_{2}$ and where the volume integral $d v$ is over the portion of three-dimensional space containing the field and includes the region where $n(\mathbf{r})>\mathbf{1}$. When $\alpha$ and $\beta$ take values $i, j=1,2,3$, we have

$$
m^{i j 0}(t)=x^{i} f^{j}-x^{j} f^{i}
$$

where $f^{i}$ are the components defined in Eq. (2.14). Equation (3.7) gives the change in total angular momentum of the field, $\Delta \mathbf{M}$, between time $t_{1}$ and $t_{2}$, and can be written as

$$
\Delta \mathbf{M}=\int\left(\mathbf{m}\left(t_{2}\right)-\mathbf{m}\left(t_{1}\right)\right) d v=\int E^{2}(\mathbf{r} \times \nabla n) d t d v
$$

where the $\mathbf{m}(t)$ is the angular momentum density at position $\mathbf{r}$ at time $t$, defined by

$$
\mathbf{m}(t)=\mathbf{r} \times \mathbf{g}_{\mathbf{G}}(t)
$$

and is defined in terms of the Gordon momentum density

$$
\mathbf{g}_{\mathbf{G}}(t)=n \mathbf{E} \times \mathbf{B} / c .
$$

Equation (3.9) shows that when $\mathbf{r} \times \nabla n$ is non-zero, then the total angular momentum of the field (as expressed through the angular momentum density) can change. Once again, we remind the reader that we have assumed the index of refraction as isotropic, constant in time, but varying in position. Equation (3.9) essentially shows that a particular spatial distribution of $\mathbf{r} \times \nabla n$ can lead to a back-action on the field that can alter the field angular momentum. Indeed this has been exploited in a number of recent experiments [13].

\section{SUMMARY}

In a previous work, we considered a dielectric medium with an index of refraction that was time-independent and spatially uniform, and the dielectric was covered with a thin gradient-index antireflection coating [5]. We found that the total energy and the Gordon momentum were conserved quantities (constant in time). In this work we have relaxed these conditions to include a dielectric medium that has a spatially varying index of refraction that is constant in time. The fact that the index of refraction is constant in time essentially means that the dielectric medium is mechanically rigid and subject to an external constraint. This constraint means that we are not dealing with a closed system. Using the continuity equations for energy density and momentum density, we derived a symmetric energy-momentum tensor for the electromagnetic field. Due to the fact that the system is not closed, the divergence of the stress-energy tensor is not zero, but equal to a generalized Helmholtz force vector that represents the constraint. Similarly, the divergence of the angular momentum density is not zero, due to the external constraint of a time independent index of refraction. We found that the total energy, Gordon momentum, and total angular momentum are not conserved because of the external constraint on time independence of the index of refraction. However, the time dependence of the total energy, Gordon momentum, and total angular momentum is related to the constraint, which is proportional to the gradient of the index of refraction.

Finally, we note that time $t$ has been renormalized to $\bar{t}=t / n(\mathbf{r})$ in the intermediate steps used to obtain the symmetric energy-momentum tensor and its continuity relation given by its four-divergence in Eq. (2.9). However, the final conservation laws of total energy, total momentum and total angular momentum, given by Eqs. (3.4), (3.3), and (3.9), respectively, are expressed in terms of the unrenormalized time $t$.
[1] N. Umow, "Ein Theorem über die Wechselwirkungen in Endlichen Entfernungen" Zeitschrift für Mathematik und Physik XIX, 97-114 (1874).

[2] J. H. Poynting, "On the transfer of energy in the electromagnetic field," Phil. Trans. 175, 343-361 (1884).

[3] O. Heaviside, Electromagnetic Theory, 3 vols. 1893-1912, multiple reprints including (Forgotten Books, 2010).

[4] J. Z. Buchwald, From Maxwell to Microphysics, (Univ of Chicago Press, 1985).

[5] M. E. Crenshaw and T. B. Bahder, "Energy-momentum tensor for the electromagnetic field in a dielectric," Opt. Comm. 284, 2460-2465 (2011).

[6] J. P. Gordon, "Radiation Forces and Momenta in Dielectric Media," Phys. Rev. A 8, 14-21 (1973).

[7] The assumption of a rigid dielectric medium does not violate relativistic principles, however, it leads to a non- zero four divergence of the stress-energy tensor for the electromagnetic field, since the electromagnetic field is not a closed system (because it is interacting with the dielectric medium).

[8] For a recent review, see: R. N. C. Pfeifer, T. A. Nieminen, N. R. Heckenberg, and H. Rubinsztein-Dunlop, "Colloquium: Momentum of an electromagnetic wave in dielectric media," Rev. Mod. Phys. 79, 1197-1216 (2007).

[9] H. Minkowski, Natches. Ges. Wiss. Göttingen, 53, (1908).

[10] L. D. Landau and E. M. Lifshitz, The Classical Theory of Fields, 4th. ed., (Elsevier, 2006).

[11] M. Abraham, Rend. Circ. Mat. Palermo 28, 1 (1909); 30, 33 (1910).

[12] J. D. Jackson, Classical Electrodynamics, 2nd ed., (Wiley, 1975). 
[13] For a recent review, see: J. P. Torres and L. Torner, Editors, Twisted Photons: Applications of Light with Orbital Angular Momentum, WILEY-VCH Verlag \& Co., Germany (2011).

[14] Y. S. Jiang, Y. T. He, and F. Li, "Electromagnetic Orbiytal Angular Momentum in Remote Sensing," PIERS
Proceedings, Moscow, Russia, 1330-1337 (2009).

[15] M. Padgett, S. M. Barnett, and R. Loudon, "The angular momentum of light inside a dielectric," J. Mod. Optics 50, 1555-1562 (2003). 